\title{
On Some Aspects of a New Class of Two-Piece Asymmetric Normal Distribution
}

\author{
C. Satheesh Kumar \\ Department of Statistics, University of Kerala, \\ Trivandrum 695 581, India \\ Email:drcsatheeshkumar@gmail.com
}

M.R. Anusree

Department of Operations, Rajagiri Business School, Kochi 682 039, India

Email: anusreemr@yahoo.co.in

Received 27 June 2016

Accepted 18 December 2017

\begin{abstract}
Through this chapter, we introduce a new class of two-piece asymmetric normal distribution suitable for asymmetric and plurimodal situations. We study some important aspects of this distribution by deriving explicit expressions for its distribution function, characteristic function, reliability measures etc. A location-scale extension of this class of distribution is considered and carried out the maximum likelihood estimation of its parameters. Further we have fitted the distribution to a real life data set for illustrating the usefulness of the model.
\end{abstract}

Keywords: Method of maximum likelihood, Plurimodality, Probability density function, Skewness, Skew normal distribution

\section{Introduction}

The term skew normal distribution (SND) refers to a parametric class of probability distribution that extends the normal distribution by an additional shape parameter which regulates skewness. The first systematic treatment of the SND in the scalar case was done by Azzalini (1985). He defined the SND as follows:

A random variable $\mathrm{X}$ is said to have skew normal distribution with skewness parameter $\quad=(\quad, \quad)$

$$
\theta \in R \quad-\infty \infty, \text { denoted by }
$$

$\operatorname{SND}(\theta)$, if its probability density function (p.d.f.) $g_{1}(x ; \theta)$ is of the following form. For $\mathrm{x} \in R$,

$$
\begin{gathered}
\theta \\
g_{1}(x ; \quad)=2 f(x) F(x)
\end{gathered}
$$

where $\mathrm{f}($.$) and \mathrm{F}($.) are respectively the p.d.f. and cumulative distribution function (c.d.f.) of a standard normal variate. The SND has been further studied by Azzalini (1986), Henze (1986), Azzalini and Dalla-Valle (1996), Branco and Dey (2001). Arnold et al. (1993) discussed an application of SND to psychometric data, Ball and Mankiw (1995) obtained the SND as a 
natural choice for the distribution of the relative price changes that influence the rate of inflation. A salient feature of the SND is that it is suitable for unimodal distributions and do not include multimodal distributions. Buccianti (2005) remarked that normal and skew normal models are not adequate to describe the situations of plurimodality. He investigated the shape of the frequency distribution of the $\log$ ratio, $\ln (\mathrm{cl}-=\mathrm{Na}+)$ whose components are related to water composition for 26 wells. Some SND related models that accommodates significant departures from unimodality have been developed in the literature. For example see Ma and Genton (2004) or Kumar and Anusree (2011a, 2013, 2014, 2015a, 2015b). Kumar and Anusree (2011b) defined an asymmetric version of the normal distribution as follows:

A random variable $\mathrm{Y}$ is said to follow the asymmetric normal distribution if its p.d.f. takes the following form. For y $\in R$,

$$
g_{2}(y ; \lambda, \alpha)=f(y)[\alpha+2(1-\alpha) F(\lambda y)]
$$

in which $\lambda \in R$ and $\alpha \in[0,1]$. A distribution with p.d.f. (2) we denoted as $A N D(\lambda, \alpha)$.

For getting more flexible asymmetric normal models, some researchers recently studied two-piece versions of skew normal distributions. For example see Kim (2005), Jamalizadeh et. al (2012), Kumar and Anusree (2013) and Salehi et. al (2013). The main objective of the present article is to introduce a two-piece version of the $A N D(\lambda, \alpha)$ as an asymmetric class of distribution suitable for tackling plurimodal situations. Throughout in this chapter we denote this class of distribution as "generalized two-piece asymmetric normal distribution (GTAND)". The paper is organized as follows. In section 2 we present the definition of GTAND and derive some of its important properties. In section 3, we discuss some concepts regarding the mode of the distribution. In section 4, we obtain expression for certain reliability measures such as failure rate, reliability function and mean residual life function of the GTAND. A location-scale extension of the GTAND is considered in section 5 and obtained some of its important properties. Further, the parameters of the GTAND are estimated by method of maximum likelihood in section 6 and a numerical illustration is given in section 7 .

We need the following shorter notation in the sequel. For any reals a, b and $\mathrm{k}$ such that $b x+k>0$

$$
\xi_{k}(a ; b)=\int_{a}^{\infty} \int_{0}^{\infty x+k} \frac{e^{-\frac{x^{2}+y^{2}}{2}}}{2 \pi} d y d x
$$

\section{Definition and Properties}

In this section, first we define a wide class of two-piece asymmetric normal distribution and discuss some of its important properties.

Definition 1. A random variable $Z$ is said to follow a two-piece asymmetric normal distribution with parameters $\lambda_{1}, \lambda_{2} \in R=(-\infty, \infty), \alpha \in[0,1]$ if its p.d.f. $h\left(z ; \lambda_{1}, \lambda_{2}, \alpha\right)$ is of the following form. For $z \in R$, 


$$
h\left(z ; \lambda_{1}, \lambda_{2}, \alpha\right)=\left\{\begin{array}{l}
f(z)\left[\alpha+\delta\left(\lambda_{1}, \lambda_{2}, \alpha\right) F\left(\lambda_{1} z\right)\right], z<0 \\
f(z)\left[\alpha+\delta\left(\lambda_{1}, \lambda_{2}, \alpha\right) F\left(\lambda_{2} z\right)\right], z \geq 0
\end{array}\right.
$$

where $\delta\left(\lambda_{1}, \lambda_{2}, \alpha\right)=2 \pi(1-\alpha)\left[\pi-\tan ^{-1}\left(\lambda_{1}\right)+\tan ^{-1}\left(\lambda_{2}\right)\right]^{-1}$.

Note that (4) is a proper probability density in the light of the following lemma.

Lemma 1. If $U$ is a standard normal variable, then for any real $\lambda$ and $k_{0}$,

$$
E\left[F\left(\lambda|U|+k_{0}\right)\right]=F\left\{\frac{k_{0}}{\sqrt{1+\lambda^{2}}}\right\}+2 \xi\left(\frac{k_{0}}{\sqrt{1+\lambda^{2}}}, \lambda\right),
$$

where for any $a \in R$ and $b>0$,

$$
\xi(a ; b)=\int_{a}^{\infty} \int_{0}^{\infty x} \frac{e^{-\frac{x^{2}+y^{2}}{2}}}{2 \pi} d y d x
$$

The distribution of a random variable $\mathrm{Z}$ with p.d.f. (4) we denoted as $\operatorname{GTAND}\left(\lambda_{1}, \lambda_{2}, \alpha\right)$. For some choices of $\lambda_{1}, \lambda_{2}$ and $\alpha$ the p.d.f. given in (4) of GTAND is plotted in Figures 1 and 2.

Clearly, the $\operatorname{GAND}\left(\lambda_{1}, \lambda_{2}, \alpha\right)$ contains the following special cases.

1. When $\lambda_{1}=\lambda, \lambda_{2}=\rho \lambda$ and $\alpha=0$, the distribution with p.d.f.(4) reduces to the two-piece skew normal distribution of Kumar and Anusree (2013)with parameters $\lambda$ and $\rho$,

2. when $\lambda_{1}=\lambda, \lambda_{2}=\lambda$, the distribution with p.d.f.(4) reduces to the skew normal distribution of Kumar and Anusree (2011b),

3. when , $\lambda_{1} \rightarrow-\infty, \lambda_{2} \rightarrow \infty$ or $\lambda_{1} \rightarrow-\infty, \lambda_{2} \rightarrow-\infty, \alpha=1$ or $\lambda_{1} \rightarrow \infty, \lambda_{2} \rightarrow \infty, \alpha=1$ or $\lambda_{1}=0, \lambda_{2}=0$ the distribution with p.d.f.(4) reduces to the standard normal distribution,

4. when $\lambda_{1}=-\lambda, \lambda_{2}=\lambda$ and $\alpha=0$, the distribution with p.d.f.(4) reduces to is the skew normal distribution of Kim (2005) and

5. when either $\lambda_{1} \rightarrow-\infty, \lambda_{2} \rightarrow-\infty, \alpha=2$ or $\lambda_{1} \rightarrow \infty, \lambda_{2} \rightarrow \infty, \alpha=0 \operatorname{TPSND}\left(\lambda_{1}, \lambda_{2}, \alpha\right)$, the distribution with p.d.f.(4) reduces to the half normal distribution. 
Result 1. If $Z$ follows $\operatorname{GTAND}\left(\lambda_{1}, \lambda_{2}, \alpha\right)$ with p.d.f. $h\left(z ; \lambda_{1}, \lambda_{2}, \alpha\right)$, then $Y_{1}=-Z$ follows $\operatorname{GTAND}\left(-\lambda_{2},-\lambda_{1}, \alpha\right)$.

Proof.For any $y_{1} \in R$, the p.d.f. $h_{1}\left(y_{1} ; \lambda_{1}, \lambda_{2}, \alpha\right)$ of $Y_{1}$ is given by

$$
\begin{aligned}
& h_{1}\left(y_{1} ; \lambda_{1}, \lambda_{2}, \alpha\right)=h\left(-y_{1} ; \lambda_{1}, \lambda_{2}, \alpha\right)\left|\begin{array}{l}
d z \\
d y_{1}
\end{array}\right| \\
& =\left\{\begin{array}{l}
f\left(-y_{1}\right)\left[\alpha+\delta\left(\lambda_{1}, \lambda_{2}, \alpha\right) F\left(-\lambda_{1} y_{1}\right)\right],-y_{1}<0 \\
f\left(-y_{1}\right)\left[\alpha+\delta\left(\lambda_{1}, \lambda_{2}, \alpha\right) F\left(-\lambda_{2} y_{1}\right)\right],-y_{1} \geq 0
\end{array}\right. \\
& =\left\{\begin{array}{l}
f\left(y_{1}\right)\left[\alpha+\delta\left(\lambda_{1}, \lambda_{2}, \alpha\right) F\left(-\lambda_{2} y_{1}\right)\right], y_{1} \leq 0 \\
f\left(y_{1}\right)\left[\alpha+\delta\left(\lambda_{1}, \lambda_{2}, \alpha\right) F\left(-\lambda_{1} y_{1}\right)\right], y_{1}>0
\end{array}\right.
\end{aligned}
$$

which shows that $Y_{1}=-Z$ follows $\operatorname{GTAND}\left(-\lambda_{2},-\lambda_{1}, \alpha\right)$.

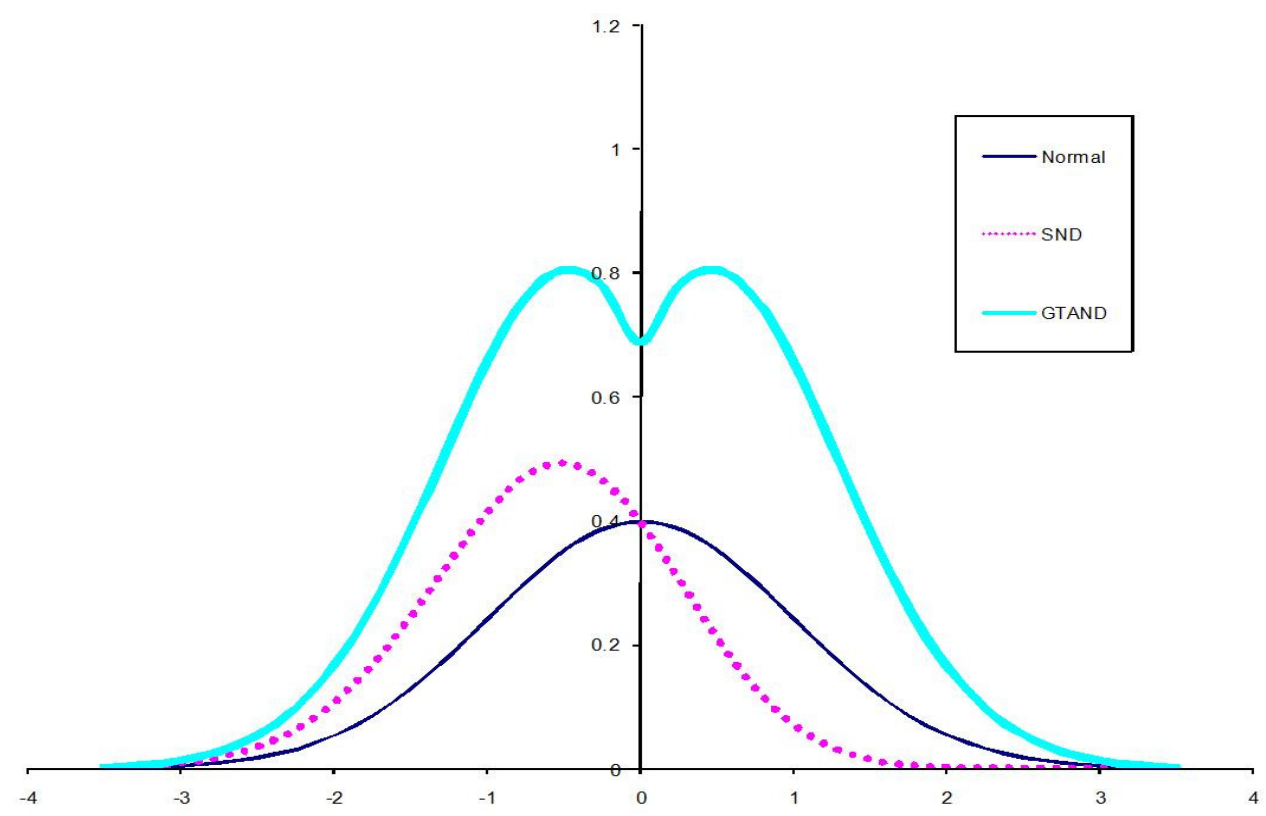

Figure1: Probability plots of Normal, SND and GTAND 


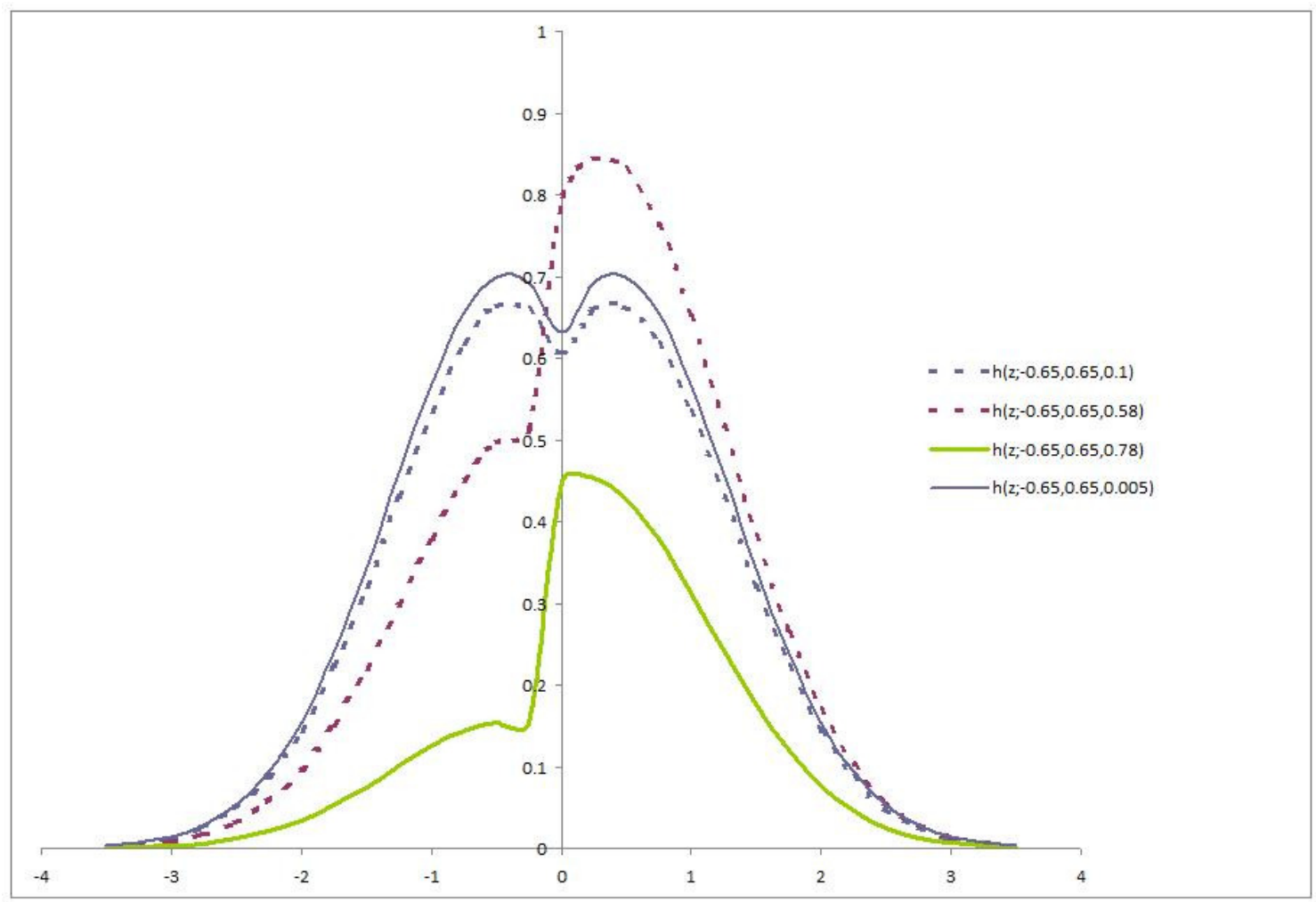

Figure2: Probability plots of $G T A N D(-0.65,0.65, \alpha)$ for different choices of $\alpha=0.1,0.58,0.78,0.005$.

Result 2. If $Z$ follows $G T A N D\left(\lambda_{1}, \lambda_{2}, \alpha\right)$ with p.d.f. $h\left(z ; \lambda_{1}, \lambda_{2}, \alpha\right)$, then $Z^{2}$ has p.d.f. (6).

Proof. The p.d.f. $h_{2}\left(y_{2} ; \lambda_{1}, \lambda_{2}, \alpha\right)$ of $Y_{2}$ is given by

$$
\begin{aligned}
& h_{2}\left(y_{2} ; \lambda_{1}, \lambda_{2}, \alpha\right)=h\left(z ; \lambda_{1}, \lambda_{2}, \alpha\right)\left|\frac{d z}{d y_{2}}\right| \\
& =h\left(-\sqrt{y_{2}} ; \lambda_{1}, \lambda_{2}, \alpha\right)\left|\frac{d z}{d y_{2}}\right|+h\left(\sqrt{y_{2}} ; \lambda_{1}, \lambda_{2}, \alpha\right)\left|\frac{d z}{d y_{2}}\right| \\
& =\frac{f\left(-\sqrt{y_{2}}\right)\left[\alpha+\delta\left(\lambda_{1}, \lambda_{2}, \alpha\right) F\left(-\lambda_{1} \sqrt{y_{2}}\right)\right]}{2 \sqrt{y_{2}}}+\frac{f\left(\sqrt{y_{2}}\right)\left[\alpha+\delta\left(\lambda_{1}, \lambda_{2}, \alpha\right) F\left(\lambda_{2} \sqrt{y_{2}}\right)\right]}{2 \sqrt{y_{2}}}
\end{aligned}
$$




$$
=\left(\frac{f\left(\sqrt{y_{2}}\right)}{2 \sqrt{y_{2}}}\right)\left[2 \alpha+\delta\left(\lambda_{1}, \lambda_{2}, \alpha\right)\left(F\left(-\lambda_{1} \sqrt{y_{2}}\right)+F\left(\lambda_{2} \sqrt{y_{2}}\right)\right)\right]
$$

Remark 1. Note that when $\lambda_{1}=0$ and $\lambda_{2}=0$, (6) reduces to the p.d.f. of a Chi-square variate with one degree of freedom.

Result 3. If $Z$ is a $\operatorname{GTAND}\left(\lambda_{1}, \lambda_{2}, \alpha\right)$ variate, then for any reals $d_{1}, d_{2}$ such that $d_{1} \leq d_{2}$,

$$
P\left(d_{1} \leq Z \leq d_{2}\right)=\left\{\begin{array}{l}
\alpha\left[F\left(d_{2}\right)-F\left(d_{1}\right)\right]+\frac{\delta\left(\lambda_{1}, \lambda_{2}, \alpha\right)}{2}\left[G\left(d_{2}, \lambda_{1}\right)-G\left(d_{1}, \lambda_{1}\right)\right], d_{1} \leq d_{2}<0 \\
\alpha\left[F\left(d_{2}\right)-F\left(d_{1}\right)\right]+\frac{\delta\left(\lambda_{1}, \lambda_{2}, \alpha\right)}{2}\left[G\left(d_{2}, \lambda_{2}\right)-G\left(d_{1}, \lambda_{2}\right)\right], 0 \leq d_{1} \leq d_{2}
\end{array}\right.
$$

where $G(., \lambda)$ is the distribution function of the $\operatorname{SND}(\lambda)$.

Proof. For any $d_{1} \leq d_{2}<0$, by definition,

$$
\begin{aligned}
& P\left(d_{1} \leq Z \leq d_{2}\right)=\int_{d_{1}}^{d_{2}} h\left(z ; \lambda_{1}, \lambda_{2}, \alpha\right) d z \\
& =\int_{d_{1}}^{d_{2}}\left[\alpha f(z)+\frac{\delta\left(\lambda_{1}, \lambda_{2}, \alpha\right)}{2} 2 f(z) F\left(\lambda_{1} z\right)\right] d z \\
& =\alpha\left[F\left(d_{2}\right)-F\left(d_{1}\right)\right]+\frac{\delta\left(\lambda_{1}, \lambda_{2}, \alpha\right)}{2}\left[G\left(d_{2}, \lambda_{1}\right)-G\left(d_{1}, \lambda_{1}\right)\right]
\end{aligned}
$$

Now, for the case $0 \leq d_{1} \leq d_{2}$,

$$
\begin{aligned}
& P\left(d_{1} \leq Z \leq d_{2}\right)=\int_{d_{1}}^{d_{2}} h\left(z ; \lambda_{1}, \lambda_{2}, \alpha\right) d z \\
& =\int_{d_{1}}^{d_{2}}\left[\alpha f(z)+\frac{\delta\left(\lambda_{1}, \lambda_{2}, \alpha\right)}{2} 2 f(z) F\left(\lambda_{2} z\right)\right] d z
\end{aligned}
$$




$$
=\alpha\left[F\left(d_{2}\right)-F\left(d_{1}\right)\right]+\frac{\delta\left(\lambda_{1}, \lambda_{2}, \alpha\right)}{2}\left[G\left(d_{2}, \lambda_{2}\right)-G\left(d_{1}, \lambda_{2}\right)\right]
$$

Thus (8) and (9) implies (7).

Result 4. The distribution function $H\left(z ; \lambda_{1}, \lambda_{2}, \alpha\right)$ of a random variable $Z$ with p.d.f. (4) is the following.

$$
H\left(z ; \lambda_{1}, \lambda_{2}, \alpha\right)=\left\{\begin{array}{l}
\alpha F(z)+\frac{\delta\left(\lambda_{1}, \lambda_{2}, \alpha\right)}{2}\left[F(z)-2 \xi\left(z, \lambda_{1}\right)\right], z<0 \\
\alpha F(z)+\frac{\delta\left(\lambda_{1}, \lambda_{2}, \alpha\right)}{2}\left[F(z)-\frac{\tan ^{-1}\left(\lambda_{1}\right)}{\pi}+\frac{\tan ^{-1}\left(\lambda_{2}\right)}{\pi}-2 \xi\left(z, \lambda_{2}\right)\right], z \geq 0
\end{array}\right.
$$

where $\xi(a ; b)$ is as defined in (5).

Proof. Let $\mathrm{Z}$ be a random variable with p.d.f. (4) and $H\left(z ; \lambda_{1}, \lambda_{2}, \alpha\right)$ be the cumulative distribution function. Then

$$
H(z)=\left\{\begin{array}{l}
L_{1}, z<0 \\
L_{2}, z \geq 0
\end{array},\right.
$$

where

$$
\begin{aligned}
& L_{1}=\int_{-\infty}^{z} \alpha f(t) d t+\frac{\delta\left(\lambda_{1}, \lambda_{2}, \alpha\right)}{2} \int_{-\infty}^{z} f(t) F\left(\lambda_{1} t\right) d t \\
& =\alpha F(z)+\frac{\delta\left(\lambda_{1}, \lambda_{2}, \alpha\right)}{2}\left\{G\left(0, \lambda_{1}\right)-\left[G\left(0, \lambda_{1}\right)-G\left(z, \lambda_{1}\right)\right]\right\} \\
& =\alpha F(z)+\frac{\delta\left(\lambda_{1}, \lambda_{2}, \alpha\right)}{2}\left(\frac{1}{2}+\frac{\tan ^{-1}\left(\lambda_{1}\right)}{\pi}\right)+\frac{\delta\left(\lambda_{1}, \lambda_{2}, \alpha\right)}{2}\left(F(z)-\frac{1}{2}-\frac{\tan ^{-1}\left(\lambda_{1}\right)}{\pi}-2 \xi\left(z, \lambda_{1}\right)\right)
\end{aligned}
$$

and 


$$
\begin{aligned}
L_{2}= & \alpha \int_{-\infty}^{z} f(t) d t+\frac{\delta\left(\lambda_{1}, \lambda_{2}, \alpha\right)}{2} \int_{-\infty}^{z} 2 f(t) F\left(\lambda_{2} t\right) d t \\
& =\int_{-\infty}^{0} \alpha f(t) d t+\delta\left(\lambda_{1}, \lambda_{2}, \alpha\right) \int_{-\infty}^{0} f(t) F\left(\lambda_{1} t\right) d t+\int_{0}^{z} \alpha f(t) d t+\delta\left(\lambda_{1}, \lambda_{2}, \alpha\right) \int_{0}^{z} f(t) F\left(\lambda_{2} t\right) d t \\
& =\alpha F(z)+\frac{\delta\left(\lambda_{1}, \lambda_{2}, \alpha\right)}{2} G\left(0, \lambda_{1}\right)+\frac{\delta\left(\lambda_{1}, \lambda_{2}, \alpha\right)}{2}\left[G\left(z, \lambda_{2}\right)-G\left(0, \lambda_{2}\right)\right] \\
= & \alpha F(z)+\frac{\delta\left(\lambda_{1}, \lambda_{2}, \alpha\right)}{2}\left(\frac{1}{2}-\frac{\tan ^{-1}\left(\lambda_{1}\right)}{\pi}\right)+\frac{\delta\left(\lambda_{1}, \lambda_{2}, \alpha\right)}{2}\left(F(z)-\frac{1}{2}+\frac{\tan ^{-1}\left(\lambda_{2}\right)}{\pi}-2 \xi\left(z, \lambda_{2}\right)\right)
\end{aligned}
$$

Now on substituting (12) and (13) in (11) we get (10).

In order to obtain the characteristic function of $\operatorname{GAND}\left(\lambda_{1}, \lambda_{2}, \alpha\right)$, we need the following lemma from Ellison (1964),

Lemma 2. For any $a_{1}, a_{2} \in R$ and a standard normal variable $Z$ with distribution function $F$

$$
E\left\{F\left(a_{1} Z+a_{2}\right)\right\}=F\left\{\frac{a_{2}}{\sqrt{\left(1+a_{1}^{2}\right)}}\right\}
$$

Result 5. The characteristic function, $\phi_{Z}(t)$ of a random variable $Z$ following $G T A N D\left(\lambda_{1}, \lambda_{2}, \alpha\right)$ with p.d.f (4) is the following, for any $t \in R$ and $i=\sqrt{-1}$.

$$
\phi_{Z}(t)=e^{-\frac{t^{2}}{2}}\left[\alpha+\delta\left(\lambda_{1}, \lambda_{2}, \alpha\right) F\left(i \delta_{1} t\right)\right]-\delta\left(\lambda_{1}, \lambda_{2}, \alpha\right) e^{-\frac{t^{2}}{2}}\left[\xi_{s_{1}}\left(-i t, \lambda_{1}\right)-\xi_{s_{2}}\left(-i t, \lambda_{2}\right)\right]
$$

where, $\delta_{1}=\frac{\lambda_{1}}{\sqrt{1+\lambda_{1}^{2}}}, s_{j}=i t \lambda_{j}$, for $\mathrm{j}=1,2$ and $\xi_{k}(a, b)$ is as defined in (3).

Proof: Let $Z$ follows $G T A N D\left(\lambda_{1}, \lambda_{2}, \alpha\right)$ with p.d.f. (4). By the definition of characteristic function, for any $t \in R$ and $i=\sqrt{-1}$, we have 
$B$ ATLANTIS

Journal of Statistical Theory and Applications, Vol. 17, No. 1 (March 2018) 101-121

$$
\begin{aligned}
\phi_{Z}(t)= & E\left(e^{i t z}\right) \\
& =\int_{-\infty}^{\infty} e^{i t z} h\left(z ; \lambda_{1}, \lambda_{2}, \alpha\right) d z \\
& =\int_{-\infty}^{\infty} e^{i t z} f(z)\left[\alpha+\delta\left(\lambda_{1}, \lambda_{2}, \alpha\right) F\left(\lambda_{1} z\right)\right] d z-\int_{0}^{\infty} e^{i t z} f(z)\left[\alpha+\delta\left(\lambda_{1}, \lambda_{2}, \alpha\right) F\left(\lambda_{1} z\right)\right] d z \\
& +\int_{0}^{\infty} e^{i t z} f(z)\left[\alpha+\delta\left(\lambda_{1}, \lambda_{2}, \alpha\right) F\left(\lambda_{2} z\right)\right] d z \\
& =e^{-t^{2}}\left\{\alpha+\delta\left(\lambda_{1}, \lambda_{2}, \alpha\right) \int_{-\infty}^{\infty} \frac{e^{-\frac{(z-i t)^{2}}{2}} F\left(\lambda_{1} z\right) d z}{\sqrt{2 \pi}} d z-\delta\left(\lambda_{1}, \lambda_{2}, \alpha\right) \int_{0}^{\infty} \frac{e^{-\frac{(z-i t)^{2}}{2}} F\left(\lambda_{1} z\right) d z}{\sqrt{2 \pi}}\right. \\
& \left.+\delta\left(\lambda_{1}, \lambda_{2}, \alpha\right) \int_{0}^{\infty} \frac{e^{-\frac{(z-i t)^{2}}{2}} F\left(\lambda_{2} z\right) d z}{\sqrt{2 \pi}}\right\}
\end{aligned}
$$

On substituting $z-i t=x, \phi_{Z}(t)$ reduces to the following.

$$
\begin{aligned}
\phi_{Z}(t)= & e^{-t^{2}}\left[\alpha+\delta\left(\lambda_{1}, \lambda_{2}, \alpha\right) \int_{-\infty}^{\infty} \frac{e^{-x^{2}} F\left(\lambda_{1}(x+i t)\right) d x}{\sqrt{2 \pi}}\right] \\
& -\delta\left(\lambda_{1}, \lambda_{2}, \alpha\right) \int_{-i t}^{\infty} \frac{e^{-x^{2}} F\left(\lambda_{1}(x+i t)\right) d x}{\sqrt{2 \pi}}+\delta\left(\lambda_{1}, \lambda_{2}, \alpha\right) \int_{-i t}^{\infty} \frac{e^{-x^{2}} F\left(\lambda_{2}(x+i t)\right) d x}{\sqrt{2 \pi}} \\
& =e^{-t^{2}}\left\{\alpha+\delta\left(\lambda_{1}, \lambda_{2}, \alpha\right) F\left(i \delta_{1} t\right)-\delta\left(\lambda_{1}, \lambda_{2}, \alpha\right) \int_{-i t}^{\infty} f(x) F\left(\lambda_{1}(x+i t)\right) d x\right. \\
& \left.+\delta\left(\lambda_{1}, \lambda_{2}, \alpha\right) \int_{-i t}^{\infty} f(x) F\left(\lambda_{2}(x+i t)\right) d x\right\}
\end{aligned}
$$

109 
in the light of Lemma 1. Thus we have

$$
\begin{aligned}
\phi_{Z}(t)= & e^{-t^{2}}\left[\alpha+\delta\left(\lambda_{1}, \lambda_{2}, \alpha\right) F(i \pi)\right]-\delta\left(\lambda_{1}, \lambda_{2}, \alpha\right) e^{-t^{2}} \int_{-i t}^{\infty} f(x)\left[\frac{1}{2}+\int_{0}^{\lambda_{1} x+s_{1}} f(u) d u\right] d x+ \\
& \delta\left(\lambda_{1}, \lambda_{2}, \alpha\right) e^{-t^{2}} \int_{-i t}^{\infty} f(x)\left[\frac{1}{2}+\int_{0}^{\lambda_{2} x+s_{2}} f(u) d u\right] d x
\end{aligned}
$$

which implies (14).

\section{Mode}

Result 6. The p.d.f. of $\operatorname{GTAND}\left(\lambda_{1}, \lambda_{2}, \alpha\right)$ is bimodal , if

(1). $\lambda_{1} \leq 0$ and $\lambda_{2} \geq 0$,

(2). $\lambda_{1} \leq 0$ or $\lambda_{2}<0$ provided and $k_{3}\left(z ; \lambda_{1}, \lambda_{2}, \alpha\right)+k_{4}\left(z ; \lambda_{1}, \lambda_{2}, \alpha\right) \leq 0$,

(3). $\lambda_{2} \geq 0$ or $\lambda_{1}>0$ provided and $k_{1}\left(z ; \lambda_{1}, \lambda_{2}, \alpha\right)+k_{2}\left(z ; \lambda_{1}, \lambda_{2}, \alpha\right) \geq 0$,

and

(4). $\lambda_{1}>0$ and $\lambda_{2}<0$ such that $k_{1}\left(z ; \lambda_{1}, \lambda_{2}, \alpha\right)+k_{2}\left(z ; \lambda_{1}, \lambda_{2}, \alpha\right) \geq 0$ and

$$
k_{3}\left(z ; \lambda_{1}, \lambda_{2}, \alpha\right)+k_{4}\left(z ; \lambda_{1}, \lambda_{2}, \alpha\right) \leq 0
$$

where

$$
\begin{aligned}
& k_{1}\left(z ; \lambda_{1}, \lambda_{2}, \alpha\right)=\frac{\lambda_{1}^{2} z f\left(\lambda_{1} z\right)}{\left[\alpha+\delta\left(\lambda_{1}, \lambda_{2}, \alpha\right) F\left(\lambda_{1} z\right)\right]}, \\
& k_{2}\left(z ; \lambda_{1}, \lambda_{2}, \alpha\right)=\frac{\lambda_{1} \delta\left(\lambda_{1}, \lambda_{2}, \alpha\right) f^{2}\left(\lambda_{1} z\right)}{\left[\alpha+\delta\left(\lambda_{1}, \lambda_{2}, \alpha\right) F\left(\lambda_{1} z\right)\right]^{2}}, \\
& k_{3}\left(z ; \lambda_{1}, \lambda_{2}, \alpha\right)=\frac{\lambda_{2}^{2} z f(\lambda z)}{\left[\alpha+\delta\left(\lambda_{1}, \lambda_{2}, \alpha\right) F\left(\lambda_{2} z\right)\right]}
\end{aligned}
$$


and

$$
k_{4}\left(z ; \lambda_{1}, \lambda_{2}, \alpha\right)=\frac{\lambda_{2} \delta\left(\lambda_{1}, \lambda_{2}, \alpha\right) f^{2}\left(\lambda_{2} z\right)}{\left[\alpha+\delta\left(\lambda_{1}, \lambda_{2}, \alpha\right) F\left(\lambda_{2} z\right)\right]^{2}} .
$$

Proof: In order to show that there exists unimodes in regions of $z \in(-\infty, 0]$ and $z \in[0, \infty)$, it is enough to show that the second derivative of $h\left(z ; \lambda_{1}, \lambda_{2}, \alpha\right)$ is negative for all $\alpha, \lambda_{1}$ and $\lambda_{2}$ in the respective region.

For $z \in(-\infty, 0)$, we have

$$
\frac{d^{2}}{d z^{2}}\left\{\log \left[h\left(z ; \lambda_{1}, \lambda_{2}, \alpha\right)\right]\right\}=-1-\lambda_{1} \delta\left(\lambda_{1}, \lambda_{2}, \alpha\right)\left[k_{1}\left(z ; \lambda_{1}, \lambda_{2}, \alpha\right)+k_{2}\left(z ; \lambda_{1}, \lambda_{2}, \alpha\right)\right]
$$

and for $z \in[0, \infty)$, we have

$$
\frac{d^{2}}{d z^{2}}\left\{\log \left[h\left(z ; \lambda_{1}, \lambda_{2}, \alpha\right)\right]\right\}=-1-\lambda_{2} \delta\left(\lambda_{1}, \lambda_{2}, \alpha\right)\left[k_{3}\left(z ; \lambda_{1}, \lambda_{2}, \alpha\right)+k_{4}\left(z ; \lambda_{1}, \lambda_{2}, \alpha\right)\right]
$$

where $k_{j}\left(z ; \lambda_{1}, \lambda_{2}, \alpha\right)$ for $\mathrm{j}=1,2,3$, and 4 are as given in (15) to (18). Note that $f\left(\lambda_{1} z\right)$ and $F\left(\lambda_{1} z\right)$ are positive for all $z \in R$ and hence $\left[\alpha+\delta\left(\lambda_{1}, \lambda_{2}, \alpha\right) F\left(\lambda_{1} Z\right)\right]$ is positive for all $\alpha>0$. For $z<0: k_{1}\left(z ; \lambda_{1}, \lambda_{2}, \alpha\right)$ is negative always and $k_{2}\left(z ; \lambda_{1}, \lambda_{2}, \alpha\right)$ is positive or negative according as $\lambda_{1}>0(<0)$. For $\lambda_{1} \leq 0$ (19) is negative and hence the density is unimodal. For $\lambda_{1}>0$, (19) is negative if $k_{1}\left(z ; \lambda_{1}, \lambda_{2}, \alpha\right)+k_{2}\left(z ; \lambda_{1}, \lambda_{2}, \alpha\right) \leq 0$. Similarly for $z \geq 0, k_{3}(z ; \lambda, \alpha)$ is always positive and $k_{4}(z ; \lambda, \alpha)$ is positive or negative according as $\lambda_{2}>0(<0)$. For $\lambda_{2} \geq 0$, (20) is negative and thus the density is unimodal and for $\lambda_{2}<0,(20)$ is negative if $k_{3}\left(z ; \lambda_{1}, \lambda_{2}, \alpha\right)+k_{4}\left(z ; \lambda_{1}, \lambda_{2}, \alpha\right) \leq 0$. Thus the proof of the result follows.

As a consequence of Result 6 we obtain the following result.

Result 7. The p.d.f. of $\operatorname{GTAND}\left(\lambda_{1}, \lambda_{2}, \alpha\right)$ is plurimodal, if

(1) $\lambda_{1} \leq 0$ or $\lambda_{2}<0$ provided and $k_{3}\left(z ; \lambda_{1}, \lambda_{2}, \alpha\right)+k_{4}\left(z ; \lambda_{1}, \lambda_{2}, \alpha\right)>0$,

(2) $\lambda_{2} \geq 0$ or $\lambda_{1}>0$ provided and $k_{1}\left(z ; \lambda_{1}, \lambda_{2}, \alpha\right)+k_{2}\left(z ; \lambda_{1}, \lambda_{2}, \alpha\right)<0$,

and 
(3) $\lambda_{1}>0$ and $\lambda_{2}<0$ such that $k_{1}\left(z ; \lambda_{1}, \lambda_{2}, \alpha\right)+k_{2}\left(z ; \lambda_{1}, \lambda_{2}, \alpha\right)>0$ and $k_{3}\left(z ; \lambda_{1}, \lambda_{2}, \alpha\right)+k_{4}\left(z ; \lambda_{1}, \lambda_{2}, \alpha\right)<0$

\section{Reliability aspects}

Here we derive some properties of the $\operatorname{GTAND}\left(\lambda_{1}, \lambda_{2}, \alpha\right)$, which are useful in reliability studies.

Let $\mathrm{Z}$ follows $\operatorname{GTAND}\left(\lambda_{1}, \lambda_{2}, \alpha\right)$ with p.d.f.(4). Now from the definition of reliability function $R\left(t ; \lambda_{1}, \lambda_{2}, \alpha\right)$ and failure rate $r\left(t ; \lambda_{1}, \lambda_{2}, \alpha\right)$ of $\mathrm{Z}$ we obtain the following results.

Result 8. The reliability function $R\left(t ; \lambda_{1}, \lambda_{2}, \alpha\right)$ of Z following the $\operatorname{GTAND}\left(\lambda_{1}, \lambda_{2}, \alpha\right)$ is

$$
R\left(t ; \lambda_{1}, \lambda_{2}, \alpha\right)=\left\{\begin{array}{l}
1-\alpha F(t)-\frac{\delta\left(\lambda_{1}, \lambda_{2}, \alpha\right)}{2}\left[F(t)-2 \xi\left(t, \lambda_{1}\right)\right], t<0 \\
1-\alpha F(t)-\frac{\delta\left(\lambda_{1}, \lambda_{2}, \alpha\right)}{2}\left[F(t)-\frac{\tan ^{-1}\left(\lambda_{1}\right)}{\pi}+\frac{\tan ^{-1}\left(\lambda_{2}\right)}{\pi}-2 \xi\left(t, \lambda_{2}\right)\right], t \geq 0
\end{array}\right.
$$

where $\xi(t,$.$) is as defined in (5) .$

Proof follows from the definition of reliability function $R\left(t ; \lambda_{1}, \lambda_{2}, \alpha\right)=1-H\left(t ; \lambda_{1}, \lambda_{2}, \alpha\right)$ where $H\left(t ; \lambda_{1}, \lambda_{2}, \alpha\right)$ is as given in Result.4.

Result 9. The failure rate $r\left(t ; \lambda_{1}, \lambda_{2}, \alpha\right)$ of $Z$ following the $\operatorname{GTAND}(\lambda, \alpha)$ is

$$
r\left(t ; \lambda_{1}, \lambda_{2}, \alpha\right)=\left\{\begin{array}{l}
\frac{f(t)\left[\alpha+\delta\left(\lambda_{1}, \lambda_{2}, \alpha\right) F\left(\lambda_{1} t\right)\right]}{1-\alpha F(t)-\frac{\delta\left(\lambda_{1}, \lambda_{2}, \alpha\right)}{2}\left[F(t)-2 \xi\left(t, \lambda_{1}\right)\right]}, t<0 \\
\frac{f(t)\left[\alpha+\delta\left(\lambda_{1}, \lambda_{2}, \alpha\right) F\left(\lambda_{2} t\right)\right]}{1-\alpha F(t)-\frac{\delta\left(\lambda_{1}, \lambda_{2}, \alpha\right)}{2}\left[F(t)-\frac{1}{\pi} \tan ^{-1}\left(\lambda_{2}\right)+\frac{1}{\pi} \tan ^{-1}\left(\lambda_{2}\right)-2 \xi\left(t, \lambda_{2}\right)\right]}, t \geq 0
\end{array}\right.
$$

Proof follows from the definition of failure rate, $r(t ; \lambda, \alpha)=\frac{h\left(t ; \lambda_{1}, \lambda_{2}, \alpha\right)}{R\left(t ; \lambda_{1}, \lambda_{2}, \alpha\right)}$ where $R\left(t ; \lambda_{1}, \lambda_{2}, \alpha\right)$ is as defined in Result 8. Further we derive the following result regarding the mean residual life function of $\operatorname{GTAND}\left(\lambda_{1}, \lambda_{2}, \alpha\right)$.

Result 10. The mean residual life function(MRLF) $\mu\left(t ; \lambda_{1}, \lambda_{2}, \alpha\right)$ of $\operatorname{GTAND}\left(\lambda_{1}, \lambda_{2}, \alpha\right)$ is 


$$
\begin{aligned}
\mu\left(t ; \lambda_{1}, \lambda_{2}, \alpha\right)= & \frac{1}{R\left(t ; \lambda_{1}, \lambda_{2}, \alpha\right)}\left\{\frac{2 \alpha e^{-t^{2}}}{\sqrt{2 \pi}}+\delta\left(\lambda_{1}, \lambda_{1}, \lambda_{2}, \alpha\right)\left(\left[F\left(\lambda_{1} t\right)+F\left(\lambda_{1} t\right)\right] f(t)\right)\right. \\
& \left.-\frac{\delta_{1}}{\sqrt{2 \pi}}\left[1-F\left(t \sqrt{1+\lambda_{1}^{2}}\right)\right]-\frac{\delta_{2}}{\sqrt{2 \pi}}\left[1-F\left(t \sqrt{1+\lambda_{2}^{2}}\right)\right]\right\}-t
\end{aligned}
$$

in which for $\mathrm{j}=1,2 \delta_{j}=\frac{\lambda_{j}}{\sqrt{1+\lambda_{j}^{2}}}$.

Proof. By definition, the MRLF of $Z$ following the $\operatorname{TAND}(\lambda, \alpha)$ is given by

$$
\begin{aligned}
\mu\left(t, \lambda_{1}, \lambda_{2}, \alpha\right) & =E(Z-t \mid Z>t) \\
& =E(Z \mid Z>t)-t
\end{aligned}
$$

where,

$$
\begin{aligned}
& E(Z \mid Z>t)=\frac{\alpha}{R\left(t ; \lambda_{1}, \lambda_{2}, \alpha\right)} \int_{t}^{\infty} z f(z) F\left(\lambda_{1} z\right) d z+\frac{\alpha}{R\left(t ; \lambda_{1}, \lambda_{2}, \alpha\right)} \int_{t}^{\infty} z f(z) F\left(\lambda_{2} z\right) d z+ \\
& \frac{\delta\left(\lambda_{1}, \lambda_{2}, \alpha\right)}{R\left(t ; \lambda_{1}, \lambda_{2}, \alpha\right)} \int_{t}^{\infty} z f(z) F\left(\lambda_{1} z\right) d z+\frac{\delta(\lambda, \alpha)}{R\left(t ; \lambda_{1}, \lambda_{2}, \alpha\right)} \int_{t}^{\infty} z f(z) F\left(\lambda_{2} z\right) d z \\
& =\frac{1}{R\left(t ; \lambda_{1}, \lambda_{2}, \alpha\right)}\left[\frac{2 \alpha e^{-t^{2}}}{\sqrt{2 \pi}}+\delta\left(\lambda_{1}, \lambda_{2}, \alpha\right)\left(I_{1}+I_{2}\right)\right]
\end{aligned}
$$

in which

$$
\begin{aligned}
& I_{1}=-\int_{t}^{\infty} f^{\prime}(z) F\left(\lambda_{1} z\right) d z \\
& =\left[F\left(\lambda_{1} t\right) f(t)\right]-\lambda_{1} \int_{t}^{\infty} f\left(\lambda_{1} z\right) f(z) d z
\end{aligned}
$$




$$
=\left\{F\left(\lambda_{1} t\right) f(t)-\frac{\lambda_{1}\left[1-F\left(t \sqrt{1+\lambda_{1}^{2}}\right)\right]}{\sqrt{2 \pi} \sqrt{1+\lambda_{1}^{2}}}\right\}
$$

and

$$
I_{2}=\left\{F\left(\lambda_{2} t\right) f(t)-\frac{\lambda_{2}\left[1-F\left(t \sqrt{1+\lambda_{2}^{2}}\right)\right]}{\sqrt{2 \pi} \sqrt{1+\lambda_{2}^{2}}}\right\},
$$

obtained in a similar way as in (25). Now on substituting (25) and (26) in (24), we get (23).

\section{Location-scale extension}

In practical situation, the location-scale extension of $\operatorname{GTAND}\left(\lambda_{1}, \lambda_{2}, \alpha\right)$ is more relevant. So in this section, we discuss the location-scale extension of the $\operatorname{GTAND}\left(\lambda_{1}, \lambda_{2}, \alpha\right)$ and present some of its important properties similar to those we obtained for $\operatorname{GTAND}\left(\lambda_{1}, \lambda_{2}, \alpha\right)$.

Definition 2. Let $Z$ follows $\operatorname{GTAND}\left(\lambda_{1}, \lambda_{2}, \alpha\right)$, then $X=\mu+\sigma Z$ is said to have an extended generalized two-piece asymmetric normal distribution with location parameter $\mu$, scale parameter $\sigma$ and shape parameters $\lambda_{1}, \lambda_{2}$ and $\alpha$, denoted as $\operatorname{EGTAND}\left(\mu, \sigma ; \lambda_{1}, \lambda_{2}, \alpha\right)$, if its p.d.f. is given by

$$
h\left(x ; \mu, \sigma, \lambda_{1}, \lambda_{2}, \alpha\right)==\left\{\begin{array}{l}
\frac{1}{\sigma} f\left(\frac{x-\mu}{\sigma}\right)\left[\alpha+\delta\left(\lambda_{1}, \lambda_{2}, \alpha\right) F\left(\lambda_{1} \frac{x-\mu}{\sigma}\right)\right], x<\mu \\
\frac{1}{\sigma} f\left(\frac{x-\mu}{\sigma}\right)\left[\alpha+\delta\left(\lambda_{1}, \lambda_{2}, \alpha\right) F\left(\lambda_{2} \frac{x-\mu}{\sigma}\right)\right], x>\mu
\end{array}\right.
$$

in which $\mu, \sigma>0, \lambda_{1}, \lambda_{2} \in R$ and $\alpha \in[0,1]$.

Clearly, the $\operatorname{EGTAND}\left(\mu, \sigma ; \lambda_{1}, \lambda_{2}, \alpha\right)$ contains the following special cases.

1. When $\lambda_{1}=\lambda, \lambda_{2}=\rho \lambda$ and $\alpha=0$, the distribution with p.d.f. (27) reduces to the location-scale extension of the twopiece skew normal distribution of Kumar and Anusree (2013),

2. when $\lambda_{1}=\lambda, \lambda_{2}=\lambda$, the distribution with p.d.f. (27) reduces to the location-scale extension of the skew normal distribution of Kumar and Anusree (2011b), 
3. when $, \lambda_{1} \rightarrow-\infty, \lambda_{2} \rightarrow \infty$ or $\lambda_{1} \rightarrow-\infty, \lambda_{2} \rightarrow-\infty, \alpha=1$ or $\lambda_{1} \rightarrow \infty, \lambda_{2} \rightarrow \infty, \alpha=1$ or $\lambda_{1}=0, \lambda_{2}=0$ the distribution with p.d.f. (27) reduces to normal distribution with parameters $\mu$ and $\sigma$,

4. when $\lambda_{1}=-\lambda, \lambda_{2}=\lambda$ and $\alpha=0$, the distribution with p.d.f. (27) reduces to location-scale extension of the skew normal distribution of Kim (2005) and

5. when either $\lambda_{1} \rightarrow-\infty, \lambda_{2} \rightarrow-\infty, \alpha=2$ or $\lambda_{1} \rightarrow \infty, \lambda_{2} \rightarrow \infty, \alpha=0 \operatorname{TPSND}\left(\lambda_{1}, \lambda_{2}, \alpha\right)$, the distribution with p.d.f. (27) reduces to the half normal distribution with parameters $\mu$ and $\sigma$.

Result 11. The characteristic function $\phi_{X}(t)$ of a random variable $X$ following $\operatorname{EGTAND}\left(\mu, \sigma ; \lambda_{1}, \lambda_{2}, \alpha\right)$ is the following, in which for each $j=1,2$ and $S_{j}=\sigma i t \lambda_{j}$. For $i=\sqrt{-1}$ and $t \in R$,

$$
\begin{aligned}
& \phi_{X}(t)=e^{i \mu t-\frac{t^{2} \sigma^{2}}{2}}\left[\alpha+\delta\left(\lambda_{1}, \lambda_{2}, \alpha\right) F\left(i \delta_{1} \sigma\right)\right]-\delta\left(\lambda_{1}, \lambda_{2}, \alpha\right) e^{i \mu t-\frac{t^{2} \sigma^{2}}{2} *} * \\
& {\left[\xi_{S_{1}}\left(-i t \sigma, \lambda_{1}\right)-\xi_{S_{2}}\left(-i t \sigma, \lambda_{2}\right)\right]}
\end{aligned}
$$

Result 12. The distribution function $H(t)=H\left(t ; \mu, \sigma, \lambda_{1}, \lambda_{2}, \alpha\right)$ of a random variable $X$ following $\operatorname{EGTAND}\left(\mu, \sigma ; \lambda_{1}, \lambda_{2}, \alpha\right)$ is the following,

$$
H(t)=\left\{\begin{array}{l}
\alpha F\left(\frac{t-\mu}{\sigma}\right)+\frac{\delta\left(\lambda_{1}, \lambda_{2}, \alpha\right)}{2}\left[F\left(\frac{t-\mu}{\sigma}\right)-2 \xi\left(\frac{t-\mu}{\sigma}, \lambda_{1}\right)\right], t<\mu \\
\alpha F\left(\frac{t-\mu}{\sigma}\right)+\frac{\delta\left(\lambda_{1}, \lambda_{2}, \alpha\right)}{2}\left[F\left(\frac{t-\mu}{\sigma}\right)-\frac{\tan ^{-1}\left(\lambda_{1}\right)}{\pi}+\frac{\tan ^{-1}\left(\lambda_{2}\right)}{\pi}-2 \xi\left(\frac{t-\mu}{\sigma}, \lambda_{2}\right)\right], t \geq \mu
\end{array}\right.
$$

where $\xi\left(\frac{t-\mu}{\sigma}, \lambda\right)$ is as defined in (5).

Result 13. The reliability function $R(t)=R\left(t ; \mu, \sigma, \lambda_{1}, \lambda_{2}, \alpha\right)$ of a random variable $X$ following $\operatorname{EGTAND}\left(\mu, \sigma ; \lambda_{1}, \lambda_{2}, \alpha\right)$ is the following, 


$$
R(t)=\left\{\begin{array}{l}
1-\alpha F\left(\frac{t-\mu}{\sigma}\right)-\frac{\delta\left(\lambda_{1}, \lambda_{2}, \alpha\right)}{2}\left[F\left(\frac{t-\mu}{\sigma}\right)-2 \xi\left(\frac{t-\mu}{\sigma}, \lambda_{1}\right)\right], t<\mu \\
1-\alpha F\left(\frac{t-\mu}{\sigma}\right)-\frac{\delta\left(\lambda_{1}, \lambda_{2}, \alpha\right)}{2}\left[F\left(\frac{t-\mu}{\sigma}\right)-\frac{\tan ^{-1}\left(\lambda_{1}\right)}{\pi}+\frac{\tan ^{-1}\left(\lambda_{2}\right)}{\pi}-2 \xi\left(\frac{t-\mu}{\sigma}, \lambda_{2}\right)\right], t \geq \mu
\end{array}\right.
$$

where $\xi\left(\frac{t-\mu}{\sigma}, \lambda\right)$ is as defined in (5).

Result 5.4 The failure rate $r(t)=r\left(t ; \mu, \sigma, \lambda_{1}, \lambda_{2}, \alpha\right)$ of a random variable $X$ following EGTAND $\left(\mu, \sigma ; \lambda_{1}, \lambda_{2}, \alpha\right)$ is the following,

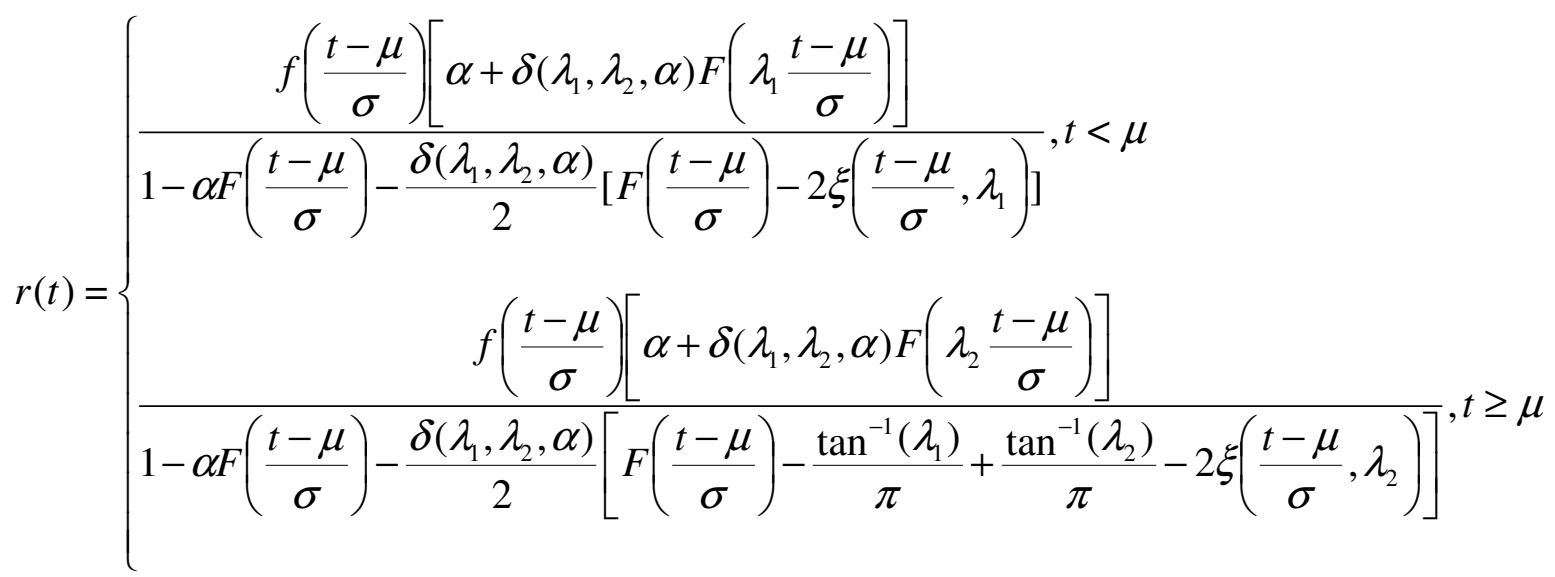

\section{Estimation}

Let $X_{1}, X_{2}, \ldots, X_{n}$ be a random sample from $\operatorname{EGTAND}\left(\mu, \sigma ; \lambda_{1}, \lambda_{2}, \alpha\right)$ with p.d.f. (27). Let $X_{(1)}, X_{(2)}, \ldots, X_{(n)}$ be the ordered sample. Assume $X_{(r)}<\mu<X_{(r+1)}$, for a particular $\mathrm{r}=1,2, \ldots, \mathrm{n}$. Then log-likelihood function of the sample is the following, in which $\Sigma_{I_{j}}$, denote the summation over the set $I_{j}$ such that

$$
\begin{array}{r}
I_{1}=\left\{i: X_{(i)}<\mu, \text { for } i=1,2, \ldots, r\right\} \text { and } I_{2}=\left\{i: X_{(i)} \geq \mu, \text { for } i=r+1, . ., n\right\} . \\
\log L=-n \ln \sigma+\sum_{I_{1}} \ln f\left(\frac{x_{i}-\mu}{\sigma}\right)\left[\alpha+\delta\left(\lambda_{1}, \lambda_{2}, \alpha\right) F\left(\frac{\lambda_{1}\left(x_{i}-\mu\right)}{\sigma}\right)\right]+
\end{array}
$$




$$
\sum_{I_{2}} \ln f\left(\frac{\lambda_{2}\left(x_{i}-\mu\right)}{\sigma}\right)\left[\alpha+\delta\left(\lambda_{1}, \lambda_{2}, \alpha\right) F\left(\frac{\lambda_{2}\left(x_{i}-\mu\right)}{\sigma}\right)\right]
$$

On differentiating (28) with respect to the parameters $\mu, \sigma, \lambda_{1}, \lambda_{2}$ and $\alpha$ and equating to zero, we obtain the following likelihood equations:

$$
\begin{aligned}
& \sum_{I_{1}}\left(\frac{x_{i}-\mu}{\sigma^{2}}\right)-\frac{\lambda_{1} \delta\left(\lambda_{1}, \lambda_{2}, \alpha\right)}{\sigma} \sum_{I_{1}} \frac{f\left(-\frac{\lambda_{1}}{\sigma}\left(x_{i}-\mu\right)\right)}{\left[\alpha+\delta\left(\lambda_{1}, \lambda_{2}, \alpha\right) F\left(\frac{\lambda_{1}}{\sigma}\left(x_{i}-\mu\right)\right)\right]} \\
& +\sum_{I_{2}}\left(\frac{x_{i}-\mu}{\sigma^{2}}\right)-\frac{\lambda_{2} \delta\left(\lambda_{1}, \lambda_{2}, \alpha\right)}{\sigma} \sum_{I_{2}} \frac{f\left(\frac{\lambda_{2}}{\sigma}\left(x_{i}-\mu\right)\right)}{\left[\alpha+\delta\left(\lambda_{1}, \lambda_{2}, \alpha\right) F\left(\frac{\lambda_{2}}{\sigma}\left(x_{i}-\mu\right)\right)\right]}=0 \\
& -\frac{n}{\sigma^{2}}+\sum_{I_{1}} \frac{\left(x_{i}-\mu\right)^{2}}{\sigma^{4}}-\sum_{I_{1}} \frac{f\left(\frac{\lambda_{1}}{\sigma}\left(x_{i}-\mu\right)\right)}{\left[\alpha+\delta\left(\lambda_{1}, \lambda_{2}, \alpha\right) F\left(\frac{\lambda_{1}}{\sigma}\left(x_{i}-\mu\right)\right)\right]}\left(x_{i}-\mu\right) \frac{\delta\left(\lambda_{1}, \lambda_{2}, \alpha\right) \lambda_{1}}{\sigma^{3}} \\
& +\sum_{I_{2}} \frac{\left(x_{i}-\mu\right)^{2}}{\sigma^{4}}-\sum_{I_{2}} \frac{f\left(\frac{\lambda_{2}}{\sigma}\left(x_{i}-\mu\right)\right)}{\left[\alpha+\delta\left(\lambda_{1}, \lambda_{2}, \alpha\right) F\left(\frac{\lambda_{2}}{\sigma}\left(x_{i}-\mu\right)\right)\right]}\left(x_{i}-\mu\right) \frac{\delta\left(\lambda_{1}, \lambda_{2}, \alpha\right) \lambda_{2}}{\sigma^{3}}=0 \\
& -\delta\left(\lambda_{1}, \lambda_{2}, \alpha\right) \sum_{I_{1}} \frac{f\left(\frac{\lambda_{1}\left(x_{i}-\mu\right)}{\sigma}\right) \frac{\left(x_{i}-\mu\right)}{\sigma}}{\left[\alpha+\delta\left(\lambda_{1}, \lambda_{2}, \alpha\right) F\left(\frac{\lambda_{1}}{\sigma}\left(x_{i}-\mu\right)\right)\right]}=0 \\
& \delta\left(\lambda_{1}, \lambda_{2}, \alpha\right) \sum_{I_{2}} \frac{f\left(\frac{\lambda_{2}\left(x_{i}-\mu\right)}{\sigma}\right) \frac{\left(x_{i}-\mu\right)}{\sigma}}{\left[\alpha+\delta\left(\lambda_{1}, \lambda_{2}, \alpha\right) F\left(\lambda_{2} \frac{\left(x_{i}-\mu\right)}{\sigma}\right)\right]}=0
\end{aligned}
$$




$$
\sum_{I_{1}} \frac{1-2 F\left(\frac{\lambda_{1}\left(x_{i}-\mu\right)}{\sigma}\right)}{\left[\alpha+\delta\left(\lambda_{1}, \lambda_{2}, \alpha\right) F\left(\frac{\lambda_{1}\left(x_{i}-\mu\right)}{\sigma}\right)\right]}+\sum_{I_{2}} \frac{1-2 F\left(\frac{\lambda_{1}\left(x_{i}-\mu\right)}{\sigma}\right)}{\left[\alpha+\delta\left(\lambda_{1}, \lambda_{2}, \alpha\right) F\left(\frac{\lambda_{2}\left(x_{i}-\mu\right)}{\sigma}\right)\right]}=0
$$

Let

$$
w\left(x_{i}\right)=\frac{f\left(\lambda_{1} \frac{\left(x_{i}-\mu\right)}{\sigma}\right)}{\left[\left[\alpha+\delta\left(\lambda_{1}, \lambda_{2}, \alpha\right) F\left(\lambda_{1} \frac{\left(x_{i}-\mu\right)}{\sigma}\right)\right]\right]},
$$

and

$$
\begin{gathered}
\Omega\left(x_{i}\right)=\frac{f\left(\lambda_{2} \frac{\left(x_{i}-\mu\right)}{\sigma}\right)}{\left[\left[\alpha+\delta\left(\lambda_{1}, \lambda_{2}, \alpha\right) F\left(\lambda_{2} \frac{\left(x_{i}-\mu\right)}{\sigma}\right)\right]\right]}, \\
W\left(x_{i}\right)=\left[\begin{array}{c}
{\left[\lambda_{1} \frac{\left(x_{i}-\mu\right)}{\sigma}\right)} \\
{\left[\alpha+\delta\left(\lambda_{1}, \lambda_{2}, \alpha\right) F\left(\lambda_{1} \frac{\left(x_{i}-\mu\right)}{\sigma}\right)\right]}
\end{array}\right]
\end{gathered}
$$

and

$$
\Delta\left(x_{i}\right)=\frac{F\left(\lambda_{2} \frac{\left(x_{i}-\mu\right)}{\sigma}\right)}{\left.\left[\alpha+\delta\left(\lambda_{1}, \lambda_{2}, \alpha\right) F\left(\lambda_{2} \frac{\left(x_{i}-\mu\right)}{\sigma}\right)\right]\right]} .
$$

Then the equations from (29) to (33) becomes

$$
\sum_{I_{1}} \frac{\left(x_{i}-\mu\right)}{\sigma}+\sum_{I_{2}} \frac{\left(x_{i}-\mu\right)}{\sigma}=\delta\left(\lambda_{1}, \lambda_{2}, \alpha\right)\left[\lambda_{1} \sum_{I_{1}} w\left(x_{i}\right)+\lambda_{2} \sum_{I_{2}} \Omega\left(x_{i}\right)\right],
$$




$$
\begin{gathered}
\frac{n}{2 \sigma^{2}}=\frac{1}{2} \sum_{I_{1}} \frac{\left(x_{i}-\mu\right)^{2}}{\sigma^{4}}+\frac{1}{2} \sum_{I_{2}} \frac{\left(x_{i}-\mu\right)^{2}}{\sigma^{4}} \\
-\frac{\delta\left(\lambda_{1}, \lambda_{2}, \alpha\right) \lambda}{2 \sigma^{3}}\left(\lambda_{1} \sum_{I_{1}} w\left(x_{i}\right)\left(x_{i}-\mu\right)+\lambda_{2} \sum_{I_{2}} \Omega\left(x_{i}\right)\left(x_{i}-\mu\right)\right), \\
\delta\left(\lambda_{1}, \lambda_{2}, \alpha\right) \sum_{I_{1}} w\left(x_{i}\right)\left(\frac{x_{i}-\mu}{\sigma}\right)=0, \\
\delta\left(\lambda_{1}, \lambda_{2}, \alpha\right) \sum_{I_{2}} \Omega\left(x_{i}\right)\left(\frac{x_{i}-\mu}{\sigma}\right)=0 .
\end{gathered}
$$

and

$$
\begin{aligned}
& 2 \sum_{I_{1}} W\left(x_{i}\right)+2 \sum_{I_{2}} \Delta\left(x_{i}\right)=\sum_{I_{1}} \frac{1}{\left.\alpha+\delta\left(\lambda_{1}, \lambda_{2}, \alpha\right) F\left(\lambda_{1} \frac{x_{i}-\mu}{\sigma}\right)\right]} \\
& +\sum_{I_{2}} \frac{1}{\left.\alpha+\delta\left(\lambda_{1}, \lambda_{2}, \alpha\right) F\left(\lambda_{2} \frac{x_{i}-\mu}{\sigma}\right)\right]}
\end{aligned}
$$

On solving the non-linear system of equations (34) to (38) by simultaneous solution method using some mathematical softwares such as MATHCAD, MATLAB, MATHEMATICA etc. one can obtain the maximum likelihood estimates (MLE) of the parameters of $\operatorname{EGTAND}\left(\mu, \sigma ; \lambda_{1}, \lambda_{2}, \alpha\right)$.

\section{Numerical computation}

For a numerical illustration,we consider the following real life data set on the heights (in centimeters) of 100 Australian athletes, given in Cook and Weisberg (1994).The data recorded is as given below.

148.9149156156 .9157 .9158 .9162162162 .5163163 .9165166 .1166 .7167 .3167 .9168168 .6169 .1169 .8169 .9170 170170.3170 .8171 .1171 .4171 .4171 .6171 .7172172 .2172 .3172 .5172 .6172 .7173173 .3173 .3173 .5173 .6173 .7173 .8 174174174174.1174 .1174 .4175175175175 .3175 .6176176176176176 .8177177 .3177 .3177 .5177 .5177 .8177 .9

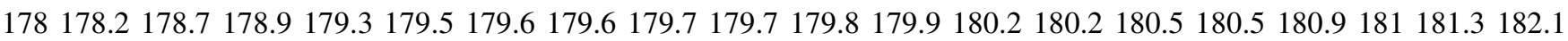
182.7183183 .3183 .3184 .6184 .7185185 .2186 .2186 .3188 .7189 .7193 .4195 .9$. 
This data has been recently used by Salehi et. al (2013) for establishing that "generalized skew two-piece skew normal distribution[ $\operatorname{GSTPSt}\left(\lambda_{1}, \lambda_{2}, \rho\right)$ ]"fits the data better than certain existing models. We obtained the MLE of the parameters of of $N(\mu, \sigma)$, the location-scale extension of $\operatorname{SND}(\lambda)$ [ $\operatorname{ESND}((\mu, \sigma ; \lambda)]$, the location-scale extension of $\operatorname{TPSND}(\lambda, \rho)$ [ $\operatorname{ETPSND}(\mu, \sigma ; \lambda, \rho)]$ of Kumar and Anusree (2013a), the location-scale extension of $\operatorname{GSTPSt}\left(\lambda_{1}, \lambda_{2}, \rho\right)$ of Salehi et. al (2013) [ $\left.\operatorname{EGSTPSt}\left(\mu, \sigma ; \lambda_{1}, \lambda_{2}, \rho\right)\right]$ and $\operatorname{EGTAND}\left(\mu, \sigma ; \lambda_{1}, \lambda_{2}, \alpha\right)$ with the help of equations (6.7) to (6.11) and MATHCAD software. The values of loglikelihood $(l)$, the Akaike's Information Criterion (AIC), the Bayesian Information Criterion (BIC) and the corrected Akaike's Information Criterion (AICc) are also computed and presented in Table 1.

Table 1. Estimated values of the parameters and the corresponding $l$, AIC, BIC and AICc values for the fitted models-the $N(\mu, \sigma)$, the $\operatorname{ESND}\left((\mu, \sigma ; \lambda)\right.$, the $\operatorname{ETPSND}(\mu, \sigma ; \lambda, \rho)$, the $\operatorname{EGSTPSt}\left(\mu, \sigma ; \lambda_{1}, \lambda_{2}, \rho\right)$ and the $\operatorname{EGTAND}\left(\mu, \sigma ; \lambda_{1}, \lambda_{2}, \alpha\right)$.

\begin{tabular}{|c|c|c|c|c|c|}
\hline Distribution: & Normal & ESND & ETPSND & EGSTPSt & EGTAND \\
\hline & $(\mu, \sigma)$ & $(\mu, \sigma, \lambda)$ & $(\mu, \sigma ; \lambda, \rho)$ & $\left(\mu, \sigma ; \lambda_{1}, \lambda_{2}, \rho\right)$ & $\left(\mu, \sigma ; \lambda_{1}, \lambda_{2}, \alpha\right)$ \\
\hline$\hat{\mu}$ & 174.594 & 174.58 & 173.657 & 167.056 & 173.01 \\
\hline$\hat{\sigma}$ & 8.24 & 8.20 & 8.21 & 7.73 & 8.48 \\
\hline$\hat{\lambda}$ & - & 0.0016 & -0.18 & $0.219,0.244$ & $-2.92,0.765$ \\
\hline$\hat{\alpha}$ & - & - & - & - & 2.28 \\
\hline$\hat{\rho}$ & - & - & 0.974 & -0.991 & - \\
\hline$l$ & -352.318 & -352.318 & -349 & -348 & -347.64 \\
\hline AIC & 708.64 & 710.64 & 706 & 706 & 703 \\
\hline BIC & 713.85 & 718.45 & 716 & 705 & 704 \\
\hline AICc & 708.76 & 710.89 & 706.49 & 706.68 & \\
\hline
\end{tabular}

From Table 1 we can see that $\operatorname{EGTAND}\left(\mu, \sigma ; \lambda_{1}, \lambda_{2}, \alpha\right)$ gives a better fit to the data given to the existing models$N(\mu, \sigma), \operatorname{ESND}\left((\mu, \sigma ; \lambda), \operatorname{ETPSND}(\mu, \sigma ; \lambda, \rho)\right.$ and $\operatorname{EGSTPSt}\left(\mu, \sigma ; \lambda_{1}, \lambda_{2}, \rho\right)$. 


\section{References}

1. B. C. Arnold, R. J. Beaver, R. A. Groeneveld and W. Q. Meeker, The non truncated marginal of a truncated bivariate normal distribution, Psychometrika, 58(3) (1993), 471-488.

2. A . Azzalini, A class of distributions which includes the normal ones, Scand. Jour. Stat., 12(1985), 171-178.

3. A . Azzalini, Further results on a class of distributions which includes the normal, Statistica, 46(1986), 199-208.

4. A. Azzalini and A. Dalla Valle, The multivariate skew-normal distribution. Biometrika, 83(4) (1996), 715-726.

5. L. Ball and Mankiw, N. G, Relative price changes as aggregate supply shocks, Quart. J. Econ, 110(1995), 161193.

6. M. D. Branco and D. K. Dey, A general class of multivariate skew-elliptical distributions, Jour. Mult. Anal., 79(1) (2001), 99-113.

7. A. Buccianti, Meaning of the $\lambda$ parameter of skew normal and log skew normal distributions in fluid geo chemistry, CODAWORK'05, October 19-21(2005), 1-15.

8. R. D. Cook and S. Weisberg, An Introduction to Regression Analysis, (1994), Wiley, New York.

9. B. Ellison, Two theorems for inference about the normal distribution with applications in acceptance sampling, Jour. Amer. Stat. Assoc., 59(1964), 89-95.

10. N. Henze, A probabilistic representation of the skew-normal distribution, Scand. Jour. Stat., 13(1986), 271-275.

11. A. Jamalizadeh, A. R. Arabpour and N. Balakrishnan, A generalized skew two-piece skew-normal distribution, Stat. Papers, 52(2) (2011), 431-446.

12. H. J. Kim, On a class of two-piece skew-normal distributions, Statistics, 39(6)(2005), 537-553.

13. C. S. Kumar and M. R. Anusree, On a generalized mixture of standard normal and skew normal distributions, Stat. Prob. Lett., 81(2011a), 1813-1821.

14. C. S. Kumar and M. R. Anusree, On some properties of an asymmetric normal distribution. Research Journal of Fatima Mata National College, Science Edition, 3(2) (2011b), 35-43.

15. C. S. Kumar and M. R. Anusree, A generalized two-piece skew normal distribution and some of its properties, Statistics, 47(6) (2013), 1370-1380 .

16. C. S. Kumar and M. R. Anusree (2014), On a modified class of generalized skew normal distribution , South African Statistical Journal, 48(1)(2014), 111-124.

17. C. S. Kumar and M. R. Anusree, On an extended version of skew generalized normal distribution and some of its properties, Communications in Statistics-Theory and Methods, 44(3)(2015a), 573-586.

18. C. S. Kumar and M. R. Anusree, On modified generalized skew normal distribution and some of its properties, Journal of Statistical Theory and Practice, 9(3)(2015b), 489-505.

19. Y. Ma and M. G. Genton, A flexible class of skew-symmetric distributions, Scand. Jour. Stat., 31(2004), 459-468.

20. M.Salehi, A. Jamalizadeh and M. Doostparast, A generalized skew two piece skew elliptical distribution, Statistical Papers(2013) (Available online). 